\title{
SILÍCIO COMO AMENIZADOR DA FITOTOXICIDADE DE ZINCO EM PLANTAS JOVENS DE Eucalyptus urophylla CULTIVADAS EM SOLUÇÃO NUTRITIVA ${ }^{1}$
}

\author{
Sheila Isabel do Carmo Pinto ${ }^{2}$, Silvio Junio Ramos 3 , Josinaldo Lopes Araujo ${ }^{4}$, Valdemar Faquin ${ }^{5}$, \\ Cândido Barreto Novais ${ }^{3}$, Krisle da Silva ${ }^{6}$ e Antonio Eduardo Furtini Neto ${ }^{5}$
}

\begin{abstract}
RESUMO - Apesar de o zinco ( $\mathrm{Zn}$ ) ser micronutriente fundamental para o crescimento e metabolismo das plantas, quando presente em níveis tóxicos no ambiente pode afetar o desenvolvimento vegetal. Entre os vários efeitos benéficos do silício ( $\mathrm{Si}$ ), cita-se sua influência na diminuição ou eliminação dos efeitos adversos de metais pesados no meio. O objetivo deste trabalho foi avaliar o efeito do Si na amenização da toxidez de $\mathrm{Zn}$ sobre o crescimento e nutrição mineral de plantas de Eucalyptus urophylla. As plantas foram cultivadas em vasos contendo $3 \mathrm{~L}$ de solução nutritiva de Clark, em esquema fatorial 6 x 2, sendo seis concentrações de $\mathrm{Zn}\left(0,2,50,150,300\right.$ e $450 \mu \mathrm{mol} \mathrm{L} \mathrm{L}^{-1}$ como $\left.\mathrm{ZnSO}_{4} 7 \mathrm{H}_{2} \mathrm{O}\right)$ e duas de Si $\left(0\right.$ e $1,78 \mathrm{mmol} \mathrm{L}^{-1}$ de Si como silicato de potássio). Após oito semanas, avaliaram-se alguns parâmetros morfológicos das plantas, produção de matéria seca, teores e utilização de nutrientes. O aumento das concentrações de Zn na solução nutritiva proporcionou maior fitotoxicidade nas raízes em relação à parte aérea. A adição do Si amenizou o efeito negativo do excesso de Zn sobre o crescimento, no entanto pouco influenciou os teores dos nutrientes avaliados nos tecidos, embora tenha proporcionado utilização mais eficiente de $\mathrm{P}, \mathrm{Ca}, \mathrm{Mg}$ e S pelas plantas de Eucalyptus urophylla.
\end{abstract}

Palavras-chave: Silicato, metal pesado e nutrição de plantas.

\section{SILICON AS ALLEVIATOR OF ZINC TOXICITY IN YOUNG Eucalyptus urophylla PLANTS GROWN IN NUTRIENT SOLUTION}

\begin{abstract}
Zn is an essential micronutrient for the growth and metabolism of plants, but when present in toxic levels in the environment, it can affect the the development of plants. Among the several beneficial effects of silicon (Si), it presents an effect in the decrease or elimination of the adverse effects of heavy metals in the environment. The objective of this work was to evaluate the effect of Si in the alleviation of Zn toxicity on the growth and mineral nutrition of Eucalyptus urophylla seedlings cultivated in nutrient solution. Seedlings of Eucalyptus urophylla were grown in pots containing $3 \mathrm{~L}$ of Clark nutrient solution, in a $6 \times 2$ factorial design, using six $\mathrm{Zn}$ concentrations $\left(0,2,50,150,300\right.$ and $450 \mu \mathrm{mol} \mathrm{L} \mathrm{L}^{-1}$ as $\left.\mathrm{ZnSO}_{4} 7 \mathrm{H}_{2} \mathrm{O}\right)$ and two of Si ( $O$ and $1.78 \mathrm{mmol} \mathrm{L}^{-1}$ of Si as potassium silicate). After eight weeks, plant morphologic traits, dry matter production and content and nutrient utilization were evaluated. The increase of $Z n$ concentrations in the nutrient solution provided higher toxicity in the roots than in the shoots. The addition of the Si alleviated the negative effect of the excess $Z n$ on plant growth, but presented little effect on the nutrient content in these tissues. The silicon addition in the nutrient solution provided a more efficient use of $P, C a, M g$ and $S$ for the Eucalyptus urophylla plants.
\end{abstract}

Keywords: Silicate, heavy metal and plant nutrition.

\footnotetext{
${ }^{1}$ Recebido em 30.03.2008 e aceito para publicação em 23.06.2009.

${ }_{2}^{2}$ Departamento de Ciências Agrárias do IFMG - Campus Bambuí. Email: <sheila.isabel@ifmg.edu.br>.

${ }^{3}$ Programa de Pós-Graduação em Ciência do Solo da Universidade Federal de Lavras (UFLA). E-mail: <silviojramos @ gmail.com> e<candidobnn@yahoo.com.br>.

${ }^{4}$ Universidade Federal de Campina Grande, UFCG, Brasil. E.mail: <josinaldo@ ccta.ufcg.edu.br>.

${ }^{5}$ Departamento de Ciência do Solo da Universidade Federal de Lavras (UFLA). E-mail: <afurtini@ dcs.ufla.br>e <faquin@dcs.ufla.br>.

${ }^{6}$ Programa de Pós-Graduação em Microbiologia Agrícola da Universidade Federal de Lavras (UFLA). E-mail: <krisle00@ yahoo.com.br>.
} 


\section{INTRODUÇÃO}

A poluição do solo com metais pesados, pela intensificação das atividades industriais e agrícolas e a urbanização, é problema crescente e responsável por sérios impactos ao ambiente, dificultando o estabelecimento da vegetação, que nesse caso não é reabilitada sem a interferência antrópica. Além da industrialização e mineração, processos como a aplicação de lodo de esgoto, rejeitos industriais, pesticidas e compostos orgânicos de lixo urbano são fontes potenciais de fornecimento antrópico de diferentes elementos que podem tornar-se tóxicos para plantas e animais. O Zn, apesar de ser um micronutriente, apresenta-se como elemento potencialmente tóxico quando presente em concentrações excessivas, podendo afetar o crescimento e metabolismo de espécies vegetais (MARSCHNER, 1995).

Uma das alternativas para a revegetação de áreas contaminadas por metais pesados é a utilização de plantas como forma de descontaminar ou fitorremediar esses solos. As espécies arbóreas, devido à capacidade de imobilizar maiores quantidades de metais nos tecidos em relação às herbáceas, apresentam grande potencial para os programas de revegetação de áreas contaminadas (MARQUES et al., 2000). Devido às características de crescimento rápido, sistema radicular bastante desenvolvido e facilidade de adaptação a condições de estresse (DELL et al., 1995), o eucalipto apresenta-se com grande potencial para emprego em programas de recuperação de áreas contaminadas por metais pesados.

Embora existam trabalhos abordando o efeito da fitotoxicidade de metais pesados sobre o crescimento e nutrição mineral em espécies arbóreas (PAIVA et al., 2000; PAIVA ET AL., 2004; SOARES et al., 2005), raros são os trabalhos que avaliaram o efeito de agentes amenizantes do estresse por metais. A utilização de substâncias que beneficiem o crescimento das plantas (VANGRONSVELD et al., 1995), atuando como agentes mitigadores da fitotoxicidade dos metais, propiciando condições que permitem o estabelecimento dos vegetais, pode auxiliar na recuperação dessas áreas contaminadas.

Várias substâncias amenizantes têm sido estudadas nas mais diversas condições, como materiais orgânicos e substâncias básicas como os silicatos. Embora não tenha sido demonstrada a essencialidade do Si (EPSTEIN e BLOOM, 2006), este influencia a absorção e translocação de vários macro e micronutrientes e frequentemente diminui ou elimina os efeitos adversos do excesso de metais no meio sobre as plantas (EPSTEIN, 1994). Neuman e Nieden (2001) verificaram em Cardaminopsis halleri que o uso de Si proporcionou amenização da toxidez de Zn, e o principal mecanismo de tolerância envolvido nesse metal foi a formação de silicatos de $\mathrm{Zn}$ em solução. Contudo, a formação de silicatos como parte do mecanismo de tolerância a metais pesados não tem sido restrita somente ao $\mathrm{Zn}$. Outros autores também têm relatado a importância do Si como agente amenizante na fitotoxicidade de metais como Al, Mn, As e Cd (BAYLIS et al., 1994; HAMMOND et al., 1995; IWASAKI et al., 2002; GUO et al., 2005; LIANG et al., 2005). Segundo Liang et al. (2007), entre os mecanismos-chave mediados pelo Si para amenização dos efeitos tóxicos dos metais pesados às plantas, tem sido citado o estímulo de sistemas antioxidantes das plantas, a complexação ou coprecipitação dos íons metálicos tóxicos com Si, a imobilização dos íons metálicos tóxicos no meio de crescimento e a compartimentalização dos metais dentro das plantas. Assim, o uso do Si, como agente amenizante, associado com uma espécie vegetal mais adaptada às condições de toxidez nesses ambientes, pode contribuir para o sucesso dos programas de recuperação dessas áreas contaminadas.

Nesse sentido, o objetivo do trabalho foi avaliar o efeito do Si na amenização da fitotoxicidade de $\mathrm{Zn}$ e nutrição mineral de plantas de Eucalyptus urophylla.

\section{MATERIAL E MÉTODOS}

O experimento foi realizado em casa de vegetação no Departamento de Ciência do Solo da Universidade Federal de Lavras. As mudas de Eucalyptus urophylla, produzidas em substrato Plantmax com cerca de 10 $\mathrm{cm}$ de altura, foram transplantadas para bandejas coletivas com capacidade para $36 \mathrm{~L}$, contendo solução nutritiva de Clark (1975) com um terço da força iônica. Após uma semana, a solução foi substituída para meiaforça iônica. A solução de cultivo foi submetida à aeração constante e o pH mantido em 5,5 \pm 0,2 pela correção com $\mathrm{NaOH}$ ou $\mathrm{HCl} 0,1 \mathrm{~mol} \mathrm{~L}^{-1}$. Após um mês de aclimatação, a solução de cultivo foi substituída por uma força iônica, quando as mudas receberam os tratamentos experimentais. As mudas foram selecionadas quanto ao vigor e transplantadas para vasos de $3 \mathrm{~L}$, sendo a parcela experimental constituída por uma planta por vaso. Os tratamentos foram distribuídos em esquema fatorial 
$6 \times 2$, sendo seis concentrações de $\mathrm{Zn}(0,2,50,150$, 300 e $450 \mathrm{mmol} \mathrm{L}^{-1}$ na forma de $\left.\mathrm{ZnSO}_{4} 7 \mathrm{H}_{2} \mathrm{O}\right)$ na ausência e presença de $\mathrm{Si}\left(0\right.$ e $1,78 \mathrm{mmol} \mathrm{L}^{-1} \mathrm{de} \mathrm{Si} \mathrm{na} \mathrm{forma} \mathrm{de}$ silicato de potássio), com correção para o K nos tratamentos sem adição de $\mathrm{Si}$. O delineamento experimental utilizado foi o inteiramente casualizado com quatro repetições. Após oito semanas, avaliaram-se a altura e o diâmetro do caule das plantas e procedeu-se à colheita destas, dividindo-as em parte aérea e raízes. Durante a colheita, a sexta folha expandida das plantas foi selecionada e escaneada, obtendo-se imagens digitais para a determinação da área foliar usando o software SIARCS. Depois de lavadas, as partes das plantas foram secas em estufa $\left(65-70^{\circ} \mathrm{C}\right)$ para determinação da matéria seca da parte aérea e raiz e os teores de macro e micronutrientes (MALAVOLTA et al., 1997). Os teores de Si foram determinados conforme Furlani e Gallo (1978). De posse dos teores, determinaram-se os respectivos acúmulos, com os quais foi obtida a eficiência de utilização (EU) de P, K, Ca, Mg e S, conforme Siddiqi e Glass (1981) $\left(\mathrm{EU}=\left[(\text { matéria seca total })^{2}(\mathrm{~g}) /\right.\right.$ acúmulo total de nutriente $(\mathrm{mg})])$.

Os resultados foram submetidos à análise de variância e regressão polinomial utilizando o programa estatístico SISVAR (FERREIRA, 1999).

\section{RESULTADOS E DISCUSSÃO}

As concentrações de Zn e Si no meio de cultivo, bem como a interação entre esses fatores, afetaram significativamente os parâmetros de crescimento das plantas de Eucalyptus urophylla, embora a interação entre esses fatores não tenha sido significativa para o diâmetro e a área foliar da sexta folha expandida. O aumento da concentração de Zn na solução exerceu efeito negativo sobre a altura, área foliar, diâmetro das plantas, matéria seca da parte aérea e raiz (Figura 1). Nas maiores concentrações de $\mathrm{Zn}$, verificou-se drástica redução no crescimento das plantas em altura (Figura 1A), as quais apresentaram folhas estreitas e retorcidas, refletindo na redução de área foliar (Figura 1C). Independentemente das concentrações de Si, o diâmetro máximo $(0,95 \mathrm{~cm})$ foi obtido na concentração de $100 \mathrm{mmol} \mathrm{L}^{-1}$ de Zn (Figura 1B). Para a área foliar da sexta folha expandida (Figura 1C), o valor máximo $\left(42,5 \mathrm{~cm}^{2}\right)$ foi obtido na concentração de $192 \mathrm{mM} \mathrm{L}^{-}$ ${ }^{1}$ de $\mathrm{Zn}$. Na presença de Si, a área foliar $\left(34,3 \mathrm{~cm}^{2}\right)$, em média, foi significativamente superior em relação os tratamentos sem $\mathrm{Si}\left(29,06 \mathrm{~cm}^{2}\right)$. Na ausência de
$\mathrm{Si}$, houve aparecimento de pontuações avermelhadas próximas às nervuras das folhas na concentração de $450 \mathrm{mmol} \mathrm{L}^{-1}$ de $\mathrm{Zn}$, sintomas semelhantes aos descritos por Fontes e Cox (1998a) em Glycine max L. sob toxicidade de Zn. De acordo com Samarakoon e Rauser (1979), a toxicidade por $\mathrm{Zn}$ pode interferir no metabolismo de carboidratos e inibir o transporte de fotoassimilados, afetando o crescimento das plantas. Entre os sintomas da toxidez de $\mathrm{Zn}$, a redução no crescimento tem sido observada com frequência (FONTES e COX, 1998b; SOARES et al., 2001).

Observa-se que a altura das plantas (Figura 1A), a produção de matéria seca da parte aérea (Figura 1D) e raízes (Figura 1E) apresentaram redução linear com o aumento da concentração de $\mathrm{Zn}$ na ausência de Si. Contudo, os mesmos parâmetros analisados na presença do Si mostraram reduções quadráticas, indicando que a presença desse elemento na solução foi capaz de amenizar os efeitos da toxicidade de $\mathrm{Zn}$, principalmente nas concentrações de 150 e $300 \mathrm{mmol} \mathrm{L}^{-1}$. A altura máxima $(43,9 \mathrm{~cm})$ apresentada pelas mudas na presença do Si foi alcançada na concentração de $129 \mathrm{mmol} \mathrm{L}^{-1}$ de $\mathrm{Zn}$, enquanto na ausência de $\mathrm{Si}$ as mudas apresentaram redução de $20 \%$ na altura $(36,8 \mathrm{~cm})$, nessa mesma concentração de Zn em solução. A produção máxima de matéria seca da parte aérea $(19,2 \mathrm{~g})$ foi obtida na concentração de 59,5 mmol L-1 de Zn na presença de Si (Figura 1D). Nessa mesma concentração de Zn, omitindo $\mathrm{Si}$, houve redução de $12,5 \%$ na produção de matéria seca da parte aérea.

Nas raízes, a toxicidade de $\mathrm{Zn}$ foi ainda mais acentuada que na parte aérea, com produção de matéria seca quase nula na maior concentração desse elemento (Figura 1E). Raízes escurecidas e pouco desenvolvidas foram os sintomas observados nas maiores concentrações de $\mathrm{Zn}\left(300\right.$ e $\left.450 \mu \mathrm{M} \mathrm{L}^{-1}\right)$. Soares et al. (2001), trabalhando com E. maculata e E. urophylla, também observaram o mesmo sintoma. De acordo com Baccouch et al. (1998), esse tipo de alteração pode ser consequência da deficiência induzida de $\mathrm{Ca}$, uma vez que o $\mathrm{Zn}$ em altas concentrações pode inibir competitivamente a absorção de Ca (MALAVOLTA et al., 1997). Portanto, sob tais condições as plantas apresentam sintomas típicos de deficiência de $\mathrm{Ca}$, dada a sua função como constituinte dos pectatos de $\mathrm{Ca}$ da lamela média na parede celular, bem como no processo de divisão celular (MARSCHNER, 1995). Contudo, neste trabalho os teores de Ca nas raízes não foram afetados

R. Árvore, Viçosa-MG, v.33, n.6, p.1005-1014, 2009 


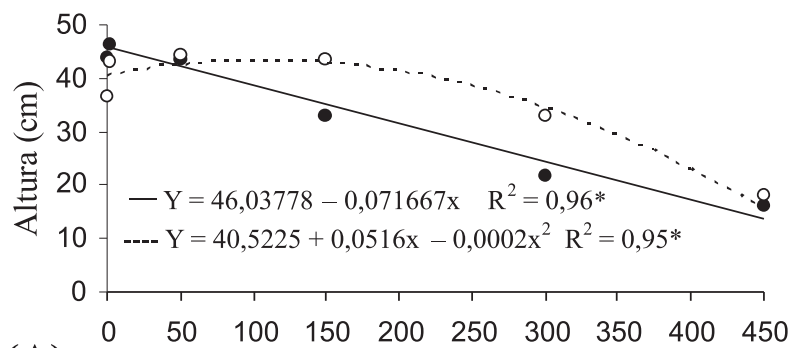

(A)
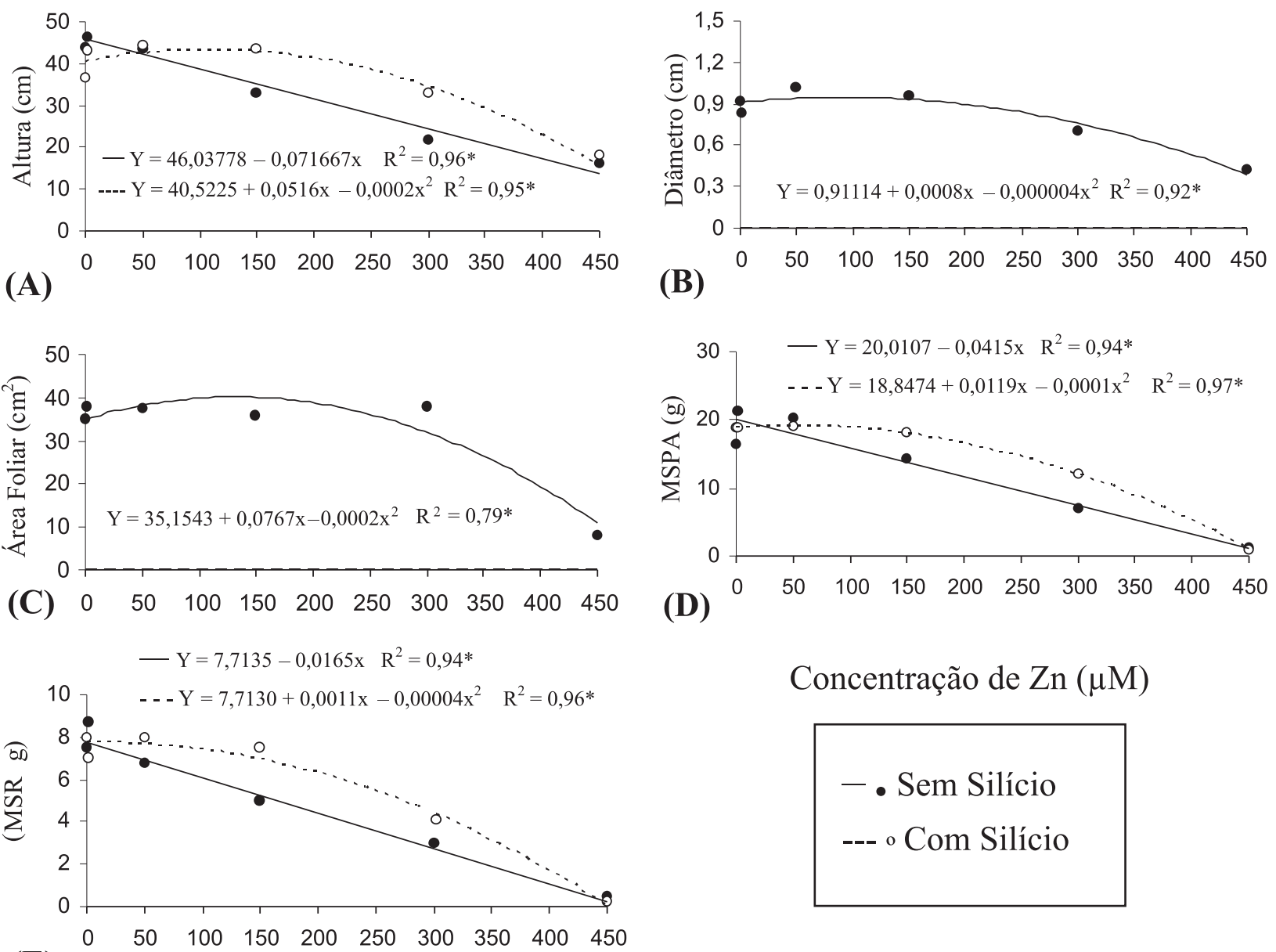

Concentração de Zn $(\mu \mathrm{M})$

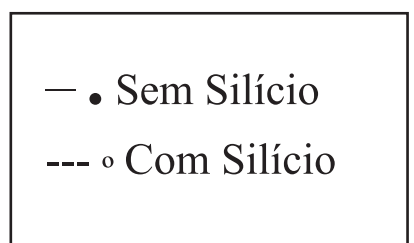

(E)

Concentração de $\mathrm{Zn}(\mu \mathrm{M})$

Figura 1 - Altura (A), diâmetro do caule (B), área foliar (C), matéria seca da parte aérea (MSPA) (D) e matéria seca da raiz (MSR) (E) de mudas de Eucalyptus urophylla em função das concentrações de Zn, na presença ou ausência de Si em solução nutritiva $(* \mathrm{pd} \leq 0,05)$.

Figure 1 - Height $(A)$, diameter of the stem $(B)$, leaf area $(C)$, shoot dry matter $(M S P A)(D)$ and root dry matter $(M S R)$ $(E)$ of Eucalyptus urophylla seedlings according to the Zn concentrations, in the presence or absence of Si in the nutrient solution $(* p d \leq 0,05)$.

significativamente pelos fatores em estudo. É importante ressaltar que, como anteriormente comentado, uma das consequências da fitotoxicidade de Zn é o decréscimo da produção de fotoassimilados e, consequentemente, a redução da translocação destes para as raízes, afetando seu crescimento e absorção de nutrientes (SAMARAKOON e RAUSER, 1979).

A máxima produção de matéria seca das raízes $(7,7$ g) foi alcançada na concentração de $13,8 \mathrm{mmol} \mathrm{L}^{-1} \mathrm{de}$ Zn na presença de Si (Figura 1E), enquanto na ausência de $\mathrm{Si}$, na mesma concentração de $\mathrm{Zn}$, verificou-se redução de apenas 2,6\% na produção de matéria seca das raízes. As doses críticas de toxidez de $\mathrm{Zn}$ na solução capazes de reduzir em $10 \%$ a altura, matéria seca da parte aérea (MSPA) e raízes (MSR) na presença de Si foram 276, 138, $154 \mathrm{mmol} \mathrm{L}^{-1}$ de Zn, respectivamente. Sem adição de $\mathrm{Si}$, esses valores decresceram acentuadamente para 64, 48 e $46 \mathrm{mmol} \mathrm{L}^{-1}$ de $\mathrm{Zn}$, respectivamente. Os valores apresentados para as doses críticas de toxicidade de Zn em solução nutritiva para a matéria seca da parte aérea e raízes foram semelhantes aos obtidos por Soares et al. (2001) em experimento sem adição de Si, em que

R. Árvore, Viçosa-MG, v.33, n.6, p.1005-1014, 2009 


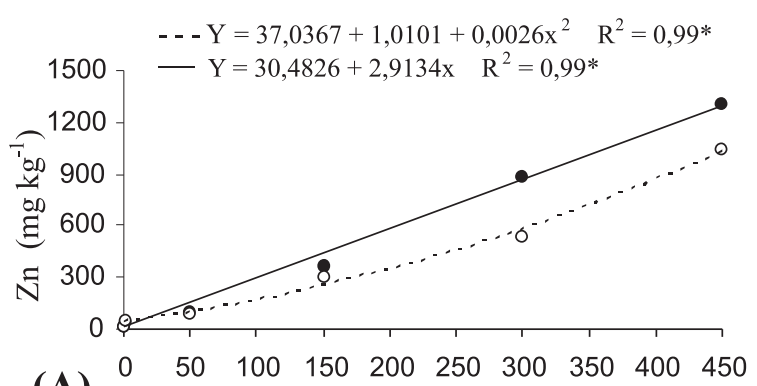

(A)

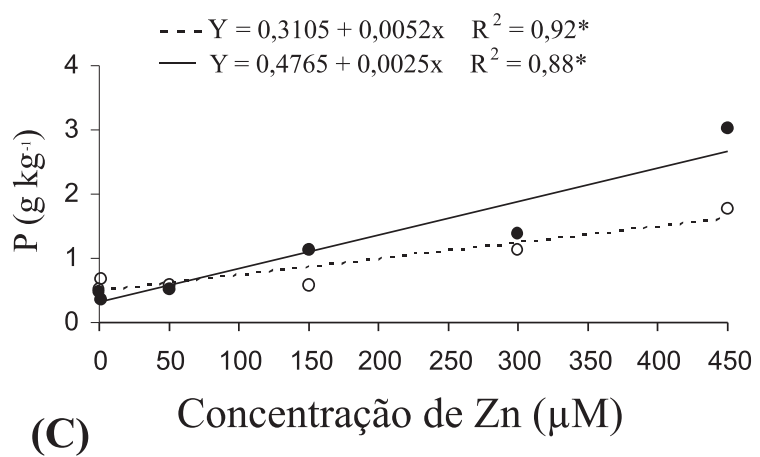

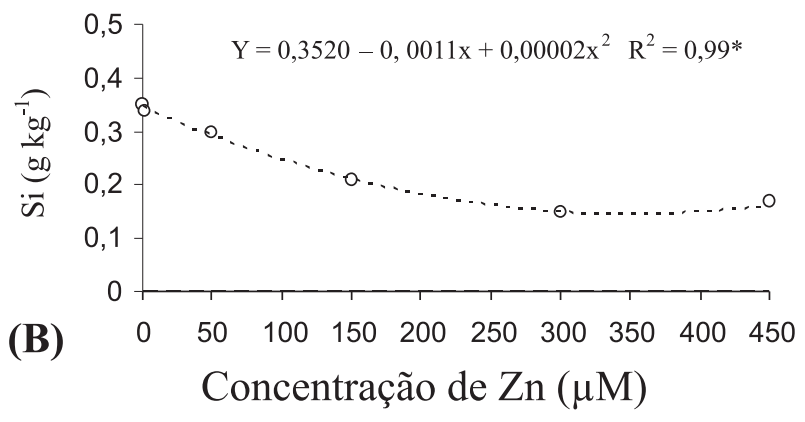

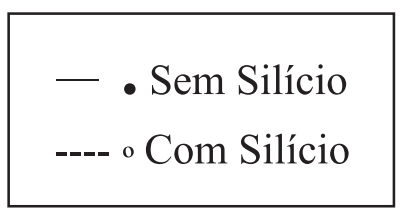

Figura 2 - Teores de Zn (A), Si (B) e P (C) na parte aérea de mudas de Eucalyptus urophylla em função das concentrações de Zn, na presença ou ausência de Si em solução nutritiva (*pd $\leq 0,05)$.

Figure 2 - Concentrations of $\mathrm{Zn}(A), \mathrm{Si}(B)$ and $P(C)$ in shoots of Eucalyptus urophylla seedlings according to the $Z n$ concentrations, in the presence or absence of Si in nutrient solution (*pd $\leq 0,05)$.

a parte aérea e raízes apresentaram concentrações críticas de 41,4 e 40,3 $\mathrm{mmol} \mathrm{L}^{-1}$ de Zn para a espécie Eucalyptus urophylla, respectivamente. Esses resultados indicam que a adição de Si na solução nutritiva pode atenuar o efeito negativo do excesso de $\mathrm{Zn}$ sobre o crescimento das plantas de eucalipto.

Os teores de Zn, Si e P na matéria seca da parte aérea (Figura 2) e os de P, K, Mg, S, Cu, Fe, Zn e Si nas raízes (Figura 3) foram influenciados significativamente pelos fatores em estudo, embora nas raízes não tenha sido verificada interação significativa entre eles para os teores de $\mathrm{K}, \mathrm{Mg}, \mathrm{Fe}$ e Si.

Na parte área, os teores de Zn na ausência de Si e os de P com ou sem a aplicação de Si aumentaram linearmente com as crescentes concentrações de $\mathrm{Zn}$ na solução (Figura 2AC). Por outro lado, os teores de Si decresceram quadraticamente com as concentrações de $\mathrm{Zn}$ na solução, mas não foram afetados significativamente na sua ausência (Figura 2B). O aumento dos teores de $\mathrm{Zn}$ na matéria seca da parte aérea se deve ao efeito combinado da absorção do
$\mathrm{Zn}$ adicionado e ao efeito de concentração dos seus teores devido ao menor crescimento das plantas em função das concentrações de Zn em solução. Para o $\mathrm{P}$, o efeito de concentração explica o aumento de seus teores em função das concentrações de $\mathrm{Zn}$. A adição de $\mathrm{Si}$, no entanto, proporcionou menores teores desses nutrientes (Figura 1AB).

A redução dos teores de $Z$ n devido à aplicação de Si também foi observada por Neuman et al. (1997). Embora não fosse possível sua comprovação neste trabalho, pôde-se levantar a hipótese de uma provável coprecipitação (NEUMANN e NIEDEN, 2001) do Zn com o Si no apoplasto radicular, uma vez que nas maiores concentrações de $\mathrm{Zn}$ os teores desse nutriente nas raízes (Figura 3G) foram maiores com a adição de $\mathrm{Si}$, enquanto na parte aérea houve diminuição da concentração de Si com as concentrações de Zn na solução. Assim, a coprecipitação mencionada diminuiria a translocação de Zn para a parte aérea. Neumam e Nieden (2001) concluíram que a formação de silicatos de $\mathrm{Zn}$ em diversos compartimentos celulares é um dos

R. Árvore, Viçosa-MG, v.33, n.6, p.1005-1014, 2009 
PINTO, S.I.C. et al.
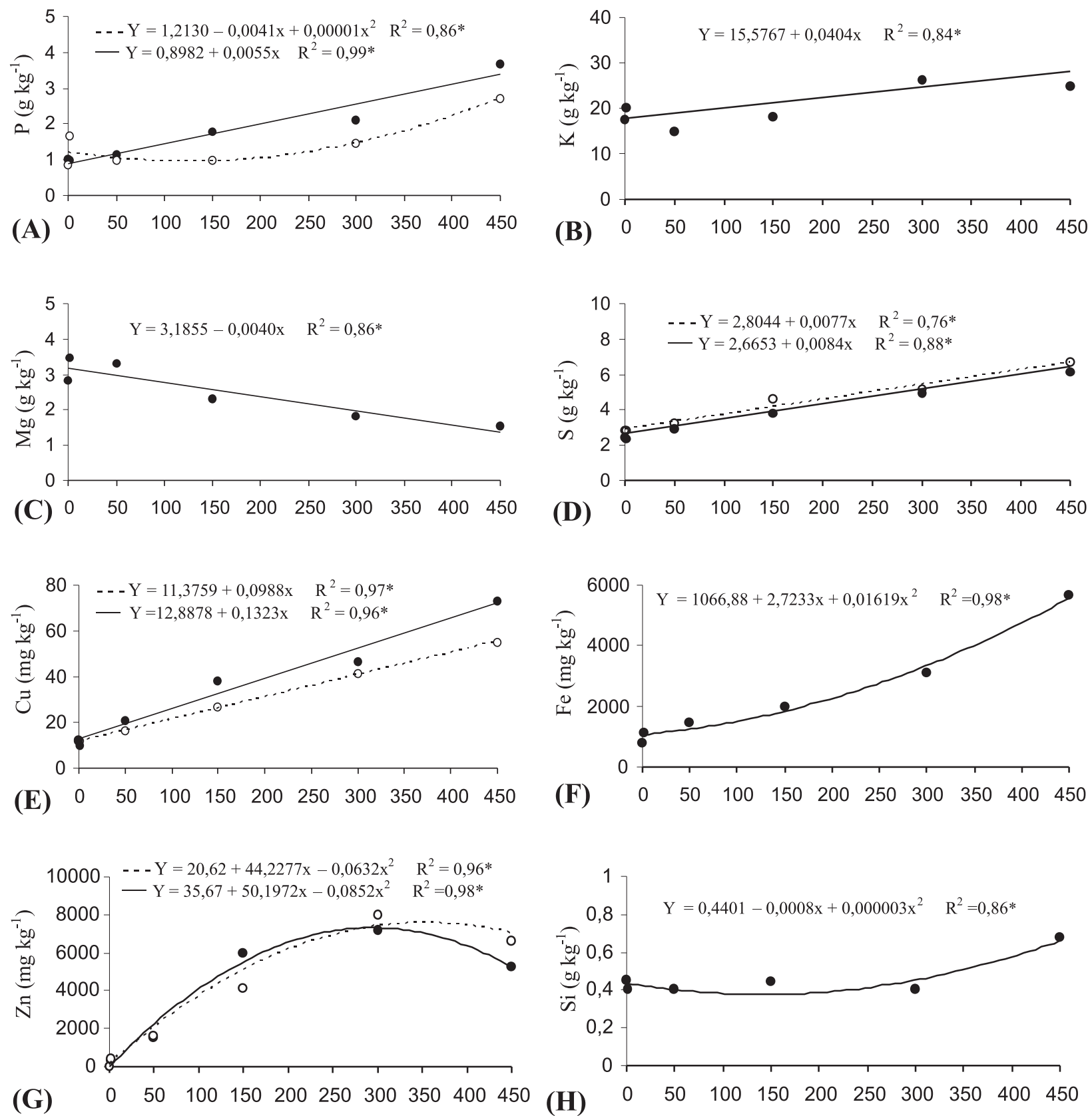

Concentração de $\mathrm{Zn}(\mu \mathrm{M})$

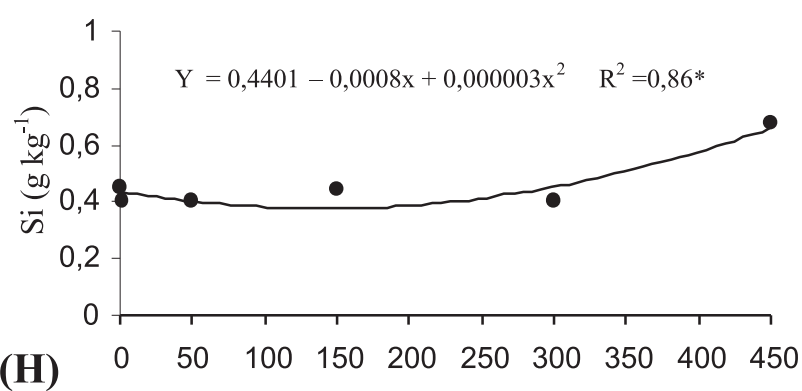

Concentração de $\mathrm{Zn}(\mu \mathrm{M})$

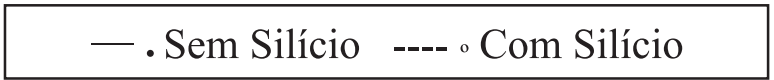

Figura 3 - Teores de P(A), K (B), Mg (C), S (D), Cu (E), Fe (F), Zn (G) e Si (H) nas raízes de mudas de Eucalyptus urophylla em função das concentrações de Zn, na presença ou ausência de Si em solução nutritiva (*pd $\leq 0,05)$.

Figure 3 - Concentrations of $P(A), K(B), M g(C), S(D), C u(E), F e(F), Z n(G)$ and $S i(H)$ in roots of Eucalyptus urophylla seedlings according to the Zn concentrations, in the presence or absence of Si in nutrient solution ( $* p d \leq 0,05)$.

R. Árvore, Viçosa-MG, v.33, n.6, p.1005-1014, 2009 
mecanismos de tolerância de Cardaminopsis halleri ao efeito tóxico desse metal. Esse efeito tem sido observado em outros trabalhos com Al (LIANG et al., 2001), Mn (WILLIAMS e VLAMIS, 1957) e Cd (LIANG et al., 2005). Além disso, a redução dos teores de Zn na parte aérea com a adição de Si pode estar também associada com o efeito de diluição, uma vez que este proporcionou maior produção de parte aérea. É importante relatar que outros mecanismos da planta mediados pelo Si podem estar envolvidos na amenização da toxicidade por metais, como o aumento da síntese de compostos fenólicos induzidos pelo
Si, os quais atuariam na complexação desses metais inativando seus efeitos tóxicos na planta (KIDD et al., 2001).

Nas raízes, os teores de todos os nutrientes avaliados, à exceção do $\mathrm{Mg}$, aumentaram com as concentrações crescentes de $\mathrm{Zn}$ adicionadas, mostrando reflexo do efeito de concentração desses elementos. No caso do Mg, houve diminuição no seu teor, o que se deve, provavelmente, à inibição competitiva no processo de absorção desse nutriente com o Zn (MARSCHNER, 1995; FAQUIN, 2005). Nesses tecidos, a adição de $\mathrm{Si}$ reduziu os teores de $\mathrm{P}$ e $\mathrm{Cu}$, enquanto
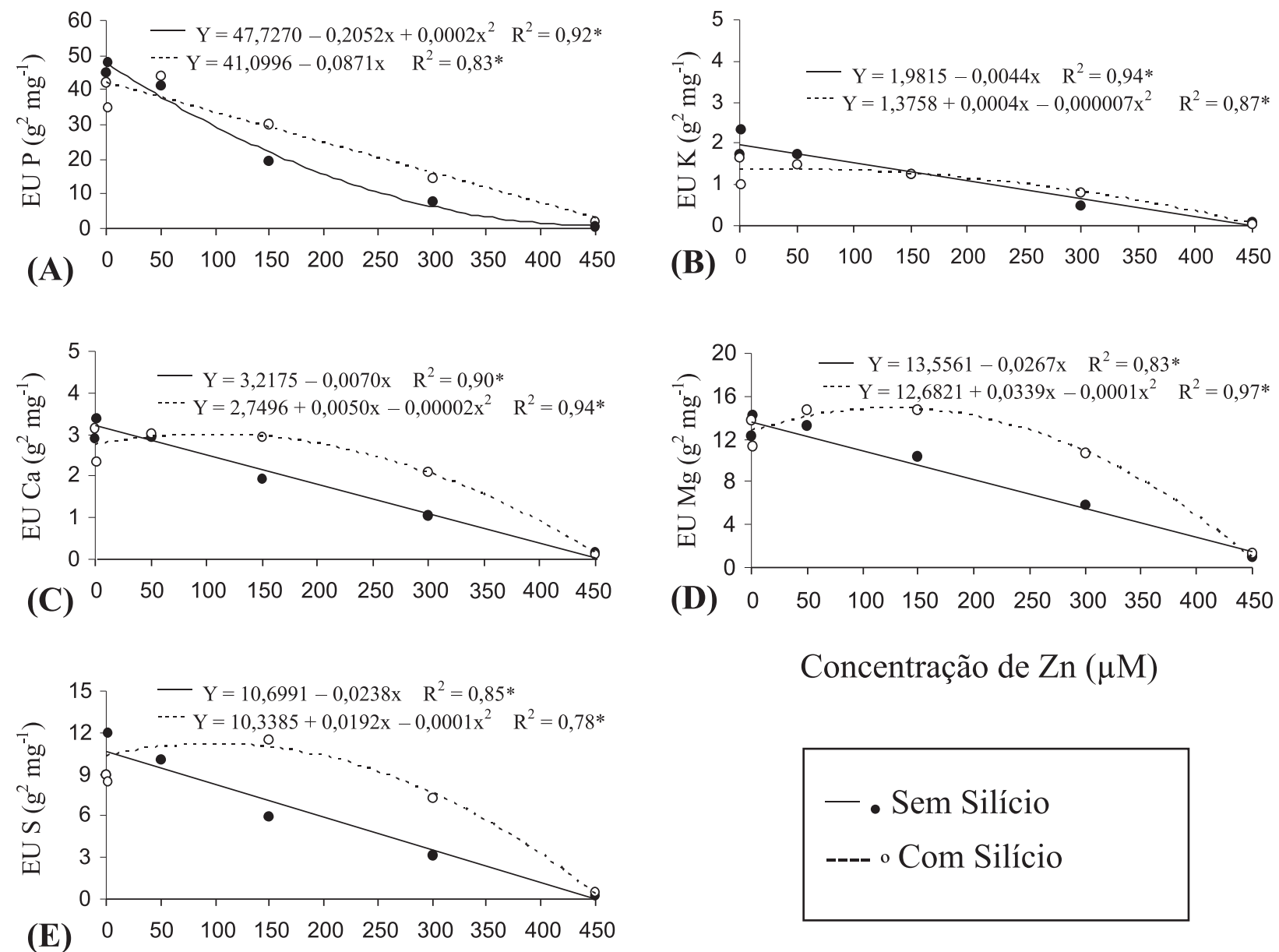

Concentração de $\mathrm{Zn}(\mu \mathrm{M})$

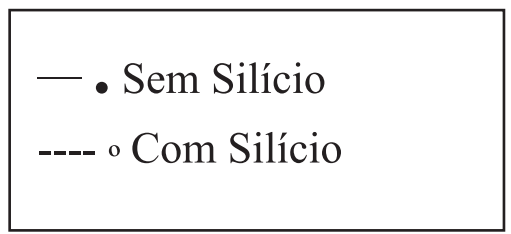

\section{Concentração de $\mathrm{Zn}(\mu \mathrm{M})$}

Figura 4 - Eficiência de utilização de P (A), K (B), Ca (C), Mg (D) e S (E) em mudas de Eucalyptus urophylla em função das concentrações de $\mathrm{Zn}$, na presença ou ausência de Si em solução nutritiva (*pd $\leq 0,05)$.

Figure 4-Efficiency of utilization of $P(A), K(B), C a(C), M g(D)$ and $S(E)$ in Eucalyptus urophylla seedlings according to the $\mathrm{Zn}$ concentrations, in the presence or absence of $S i$ in the nutrient solution $(* p d \leq 0,05)$.

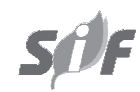

R. Árvore, Viçosa-MG, v.33, n.6, p.1005-1014, 2009 
para o $\mathrm{Zn}$ o oposto ocorreu nas concentrações acima de $300 \mu \mathrm{mol} \mathrm{L}^{-1}$ de Zn (Figura 3AEG). Na ausência de $\mathrm{Si}$, observou-se aumento de 2,8 e 4,5 vezes na concentração de $450 \mathrm{mM} \mathrm{L}^{-1}$ de $\mathrm{Zn}$ em relação à menor concentração para $\mathrm{Zn}$ e Cu, respectivamente, enquanto na presença de Si o aumento foi de apenas 1,2 e 3,9 vezes, respectivamente, para $\mathrm{Zn}$ e Cu. O teor de Si nas raízes apresentou ajuste quadrático, aumentando em função das concentrações de Zn. Esse nutriente apresentou aumento de 1,6 vezes na concentração de $450 \mathrm{mM} \mathrm{L}^{-1}$ de $\mathrm{Zn}$. Na ausência de $\mathrm{Si}$, o teor médio desse nutriente nas raízes foi de $0,22 \mathrm{~g} \mathrm{~kg}^{-1}$, enquanto na presença de $\mathrm{Si}$, de $0,71 \mathrm{~g} \mathrm{~kg}^{-1}$ (Figura $2 \mathrm{H}$ ).

A diminuição dos teores de $\mathrm{P}$ com a adição de Si também foi observada por Ma e Takahashi (1990). Esse fato poderia ser explicado pelo efeito de diluição relatado para a parte aérea (Figura 2C), entretanto esse efeito não ocorreu para o S, que apresenta concentrações comparáveis aos de P. Portanto, é provável que tenha ocorrido interação negativa do Si com o $\mathrm{P}$ no processo de absorção. Para $\mathrm{Cu}$, o efeito de diluição explicaria melhor seu comportamento devido à sua baixa concentração na solução.

A eficiência de utilização (EU) de P, K, Ca, Mg e $\mathrm{S}$ (Figura 4) foi influenciada pela interação entre as doses de Zn e a presença de Si. A EU de Ca, Mg e $\mathrm{S}$, na presença de $\mathrm{Si}$, apresentou ajuste quadrático, alcançando o valor máximo de 3,$1 ; 15,6$; e 11,3 g $\mathrm{gg}^{-}$ ${ }^{1}$, nas concentrações de $125 ; 169,5$; e $96,0 \mu \mathrm{M} \mathrm{L}^{-1} \mathrm{de}$ Zn, respectivamente, para Ca, Mg e S (Figura 4C-E). Nas mesmas concentrações de $\mathrm{Zn}$ na solução em que foram alcançadas a máxima EU na presença de $\mathrm{Si}$, observou-se uma EU de 2,3; 9,0; e 8,4 g $\mathrm{gg}^{-1}$ nos Ca, $\mathrm{Mg}$ e S, respectivamente. A EU de K, assim como ocorreu para os seus teores nas raízes (Figura 3B), pouco foi afetada pela presença do Si (Figura 4B).

$\mathrm{Na}$ ausência de $\mathrm{Si}$, a EU desses nutrientes reduziu-se linearmente com as concentrações crescentes de Zn. Esse comportamento é o resultado da menor produção de matéria das plantas e o consequente aumento nos seus teores pelo efeito de concentração. Isso ocorre porque, mesmo em condições adversas de crescimento, a planta continua absorvendo os nutrientes sem, contudo, convertê-los em produção de matéria seca. Assim, o Si parece ter sido fundamental no uso eficiente desses macronutrientes, principalmente $\mathrm{Ca}, \mathrm{Mg}$ e $\mathrm{S}$ o que em parte está relacionado com a maior produção de matéria seca na presença do Si devido ao seu efeito amenizador da toxicidade de Zn anteriormente discutido. Embora neste trabalho tenha sido evidenciado o efeito benéfico do Si nas plantas de Eucalyptus urophylla como atenuador dos efeitos negativos do excesso de $\mathrm{Zn}$, é importante ressaltar a importância de um estudo mais aprofundado no campo da fisiologia vegetal para avaliar, com maior precisão, os mecanismos envolvidos.

\section{CONCLUSÕES}

O aumento das concentrações de $\mathrm{Zn}$ na solução nutritiva reduziu o crescimento das plantas de Eucalyptus urophylla, proporcionando sintomas típicos de fitotoxicidade de Zn.

A adição do Si amenizou o efeito negativo do excesso de Zn sobre o crescimento das plantas de Eucalyptus urophylla, no entanto pouco influenciou os teores dos nutrientes avaliados nos tecidos.

A adição de Si à solução nutritiva proporcionou a utilização mais eficiente de $\mathrm{P}, \mathrm{Ca}, \mathrm{Mg}$ e S pelas plantas de Eucalyptus urophylla.

\section{REFERÊNCIAS}

BACCOUCH, S.; CHAOUI, A.; EL FERJANI, E. Nickel toxicity: effects on growth and metabolism of maize. Journal of Plant Nutrition, v.21, n.1, p.577-588, 1998.

BAYLIS, A. D. et al. Effect of silicon on the toxicity of aluminium in soybean. Comm. Soil Sci. Plant Anal., v.25, n.5, p.537-546, 1994.

CLARK, R. B. Characterization of phosphates in intact maize roots. Journal of Agricultural and Food Chemistry, v.23, n.3, p.458-460, 1975.

DELL, B.; MALAJCZUK, N.; GROVE, T. S. Nutrient disorders in plantation eucalypts. Canberra, BPD Graphic Associates, 1995.104p.

EPSTEIN, E.; BLOOM, J. A. Nutrição mineral de plantas: Princípios e perspectivas. Londrina: Planta, 2006. 403p.

EPSTEIN, E. The anomaly of silicon in plant biology. Proceedings of the National Academy of Science $\boldsymbol{U S A}$, v.91, n.1, p.11-17, 1994. 
FAQUIN, V. Nutrição mineral de mlantas. Lavras: FAEPE, 2005. 183p.

FERREIRA, D. F. SISVAR 4.3 - Sistema de análises estatísticas. Lavras: Universidade Federal de Lavras, 1999.

FONTES, R. L. F.; COX, R. Iron deficiency and zinc toxicity in soybean grown in nutrient solution with different levels of sulfur. Journal of Plant Nutrition, v.21, n.8, p.1715-1722, 1998 a.

FONTES, R. L. F.; COX, R. Zinc toxicity in soybean grown at high iron concentration in nutrient solution. Journal of Plant Nutrition, v.21, n.8, p.1723-1730, 1998b.

FURLANI, P. R.; GALLO, J. R. Determinação de silício em material vegetal, pelo método colorimétrico do "Azul-de-molibdênio". Bragantia, v.37, p. 5-11, 1978.

GUO, W. et al. Effect of silicate on the growth and arsenate uptake by rice (Oriza sativa L.) seedlings in solution culture. Plant Soil, v.272, n.1, p.173-181, 2005.

HAMMOND, K. E.; EVANS, D. E.; HODSON, M. $\mathrm{J}$. Aluminium/silicon interactions in barley (Hordeum vulgare L.) seedlings. Plant Soil, v.173, n.1, p.89-95, 1995.

IWASAKI, K. et al. Effects of silicon supply on apoplastic manganese concentrations in leaves and their relation to manganese tolerance in cowpea (Vigna unguilata (L.) Walp.). Plant Soil, v.238, n.1, p.281-288, 2002.

KIDD, P. S. et al. The role of root exudates in aluminium resistance and silicon-induced amelioration of aluminium toxicity in three varieties of maize (Zea mays L.). J. Exper. Bot, v.52, n.359, p.1339-1352, 2001.

LIANG, Y.; YANG, C.; SHI, H. Effects of silicon on growth and mineral composition of barley grown under toxic levels of aluminum. Journal of Plant Nutrition, v.24, n.2, p. 229-243, 2001.

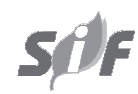

LIANG, Y. C.; WONG, J. W. C.; WEI, L. Siliconmediated enhancement of cadmium tolerance in maize (Zea mays L.) grown in cadmium contaminated soil. Chemosphere, v.58, n.4, p.475-483, 2005.

LIANG, Y. et al. Mechanisms of silicon-mediated alleviation of abiotic stresses in higher plants: A review. Environmental Pollution, v.147, p.422-428, 2007.

MA, J.; TAKAHASHI, E. Effect of silicon on the growth and phosphorus uptake of rice. Plant and Soil, v.126, n.1, p.115-119, 1990.

MALAVOLTA, E.; VITTI, G. C.; OLIVEIRA, S. A. Avaliação do estado nutricional das plantas: princípios e aplicações. 2.ed. Piracicaba, Potafos, 1997.319p.

MARQUES, T. C. L. L. S. M.; MOREIRA, F. M. S.; SIQUEIRA, J. O. Crescimento e teor de metais pesados de mudas de espécies arbóreas cultivadas em solo contaminado com metais pesados. Pesquisa Agropecuária Brasileira, v.35, n.1, p.121-132, 2000.

MARSCHNER, H. Mineral nutrition of higher plants. 2.ed. San Diego: Academic Press, 1995. 902p.

NEUMANN, D.; NIEDEN, U. Silicon and metal tolerance of higher plants. Phytochemistry, v.56, n.7, p.685-692, 2001.

NEUMANN, D. et al.. Heavy metal tolerance of Minuartia verna. Journal of Plant

Physiology, v.151, n.1, p.101-108, 1997.

PAIVA, H. N.; CARVALHO, J. G.; SIQUEIRA, J. O Efeito de $\mathrm{Cd}, \mathrm{Ni}, \mathrm{Pb}$ e $\mathrm{Zn}$ sobre mudas de cedro (Cedrella fissilis Vell.) e de ipê-roxo (Tabebuia impetiginosa (Mart.) Standley) em solução nutritiva. Revista Árvore, v.24, n.4, p.369-378, 2000 .

PAIVA, H. N. et al. Absorção de nutrientes por mudas de ipê-roxo (Tabebuia impetiginosa (Mart.) Standl.) em solução nutritiva contaminada por cádmio. Revista Árvore, v.28, n.2, p.189197, 2004.

R. Árvore, Viçosa-MG, v.33, n.6, p.1005-1014, 2009 
SAMARAKOON, A. B.; RAUSER, W.

Carbohydrate level and photoassimilate export from leaves of Phaseolus vulgaris exposed to excess cobalt, nickel and zinc. Plant

Physiology, v.63, n.6, p.1165-1169, 1979.

SIDDIQI, M. Y.; GLASS, A. D. M. Utilization index: a modified approach to the estimation and comparison of nutrient utilization efficiency in plants. Journal of Plant Nutrition, v.4, n.1, p.289-302, 1981.

SOARES. C. R. F. S. et al. Fitotoxidez de cádmio para Eucalyptus maculata e E. urophylla em solução nutritiva. Revista Árvore, v.29, n.2, p.175-183, 2005.
SOARES, C. R. F. S. et al. Toxidez de zinco no crescimento e nutrição de Eucalyptus maculata e Eucalyptus urophylla em solução nutritiva. Pesquisa Agropecuária Brasileira, v.36, n.2, p.339-348, 2001.

VANGRONSVELD, J.; van ASSCHE, F.;

CLIJSTERS, H. Reclamation of a bare industrial area contaminated by nonferrous metals: in situ metal immobilization and revegetation. Environ. Pollut., v.87, n.1, p.51-59, 1995.

WILLIAMS, D. E.; VLAMIS, J. The effect of silicon on yield and manganese-54 uptake and distribution in the leaves of barley plants grown in culture solutions. Plant Physiology, v.32, n.5, p.404-409, 1957.

R. Árvore, Viçosa-MG, v.33, n.6, p.1005-1014, 2009 